PROCEEDINGS OF THE

AMERICAN MATHEMATICAL SOCIETY

Volume 138, Number 9, September 2010, Pages 3135-3145

S 0002-9939(10)10347-5

Article electronically published on March 24, 2010

\title{
ON THE UNIVERSAL ENVELOPING ALGEBRA OF A LIE ALGEBROID
}

\author{
I. MOERDIJK AND J. MRČUN
}

(Communicated by Gail R. Letzter)

\begin{abstract}
We review the extent to which the structure of the universal enveloping algebra of a Lie algebroid over a manifold $M$ resembles a Hopf algebra, and prove a Cartier-Milnor-Moore theorem for this type of structure.
\end{abstract}

The concept of a Lie algebroid unifies many types of infinitesimal structures which occur naturally in different areas of geometry. Foliated manifolds, Poisson manifolds, Lie algebras and infinitesimal actions of Lie algebras on manifolds, for example, can all be viewed as Lie algebroids. Many Lie algebroids (but not all of them) arise as infinitesimal parts of Lie groupoids, just as finite-dimensional Lie algebras do from Lie groups.

The universal enveloping algebra of a Lie algebra is a Hopf algebra, and one recovers the Lie algebra by considering the primitive elements of the associated universal enveloping algebra. Furthermore, various forms of the Cartier-Milnor-Moore theorem give us equivalences between categories of Lie algebras and categories of cocommutative Hopf algebras.

The universal enveloping algebra of a Lie algebroid over $M$ is not a Hopf algebra in general, but it has a structure known in the literature as a $C^{\infty}(M) / \mathbb{R}$-bialgebra. In this note, we extend the Cartier-Milnor-Moore theorem from Lie algebras to Lie algebroids. Our result also extends a result of Nichols [15] which covers the special case of $K / k$-bialgebras for a field extension $K / k$. Our proof is different from the one of Nichols and is based on an extension to rings of some standard results for cocommutative coalgebras over fields, which we treat in Appendix A. It would be interesting to know whether the general results on triples of operads [7 can be extended to coloured operads so as to include our Cartier-Milnor-Moore theorem for Lie algebroids.

\section{LiE ALGEBroids AND $(k, R)$-Lie AlgEBraS}

In this section we will briefly recall the definition of Lie algebroids and mention some basic examples (see [9, 12] for more details, examples and historical remarks). We also review the construction of the universal enveloping algebra of a Lie algebroid and describe its universal property [17, 20].

Received by the editors September 28, 2009 and, in revised form, December 17, 2009.

2010 Mathematics Subject Classification. Primary 17B35, 16T10, 16T15.

The second author was supported in part by the Slovenian Research Agency (ARRS) project J1-2247.

(C)2010 American Mathematical Society 
Let $M$ be a smooth manifold. Denote by $T(M)$ the tangent bundle of $M$ and by $\mathfrak{X}(M)$ the Lie algebra of (smooth) vector fields on $M$. A Lie algebroid over $M$ is a real smooth vector bundle $\pi: \mathfrak{g} \rightarrow M$ over $M$, together with a (smooth) map an : $\mathfrak{g} \rightarrow T(M)$ of vector bundles over $M$ (called the anchor) and a Lie algebra structure on the vector space $\Gamma \mathfrak{g}$ of (smooth) sections of $\mathfrak{g}$, such that

(i) the induced map $\Gamma(\operatorname{an}): \Gamma \mathfrak{g} \rightarrow \mathfrak{X}(M)$ is a Lie algebra homomorphism, and

(ii) the Leibniz identity

$$
[X, f Y]=f[X, Y]+\Gamma(\operatorname{an})(X)(f) Y
$$

holds for any $X, Y \in \Gamma \mathfrak{g}$ and any $f \in C^{\infty}(M)$.

We write $\Gamma(\operatorname{an})(X)(f)=X(f)$. The Lie algebroids over $M$ form a category

$$
\text { (LieAlgebroids) }_{M} \text {, }
$$

where a morphism $\psi: \mathfrak{g} \rightarrow \mathfrak{g}^{\prime}$ is a map of vector bundles over $M$ intertwining the anchors (i.e. $\mathrm{an}^{\prime} \circ \psi=$ an) such that the induced map $\Gamma \psi: \Gamma \mathfrak{g} \rightarrow \Gamma \mathfrak{g}^{\prime}$ is a morphism of Lie algebras.

Example 1.1. (1) A finite-dimensional Lie algebra is a Lie algebroid over a point.

(2) The tangent bundle $T(M)$ is a Lie algebroid over $M$ with the identity map as its anchor.

(3) A foliation $\mathcal{F}$ on $M$ is an integrable subbundle of $T(M)$ and therefore a Lie algebroid over $M$ with the inclusion $\mathcal{F} \rightarrow T(M)$ as its anchor.

(4) The right invariant vector fields on a Lie groupoid over a manifold $M$ (cf. [9, 12]) correspond to sections of a vector bundle over $M$ which is a Lie algebroid. The anchor map of this Lie algebroid is induced by the target map of the Lie groupoid. A Lie algebroid over $M$ is integrable if it is isomorphic to the Lie algebroid associated to a Lie groupoid. There is an equivalence between the category of integrable Lie algebroids over $M$ and the category of Lie groupoids over $M$ with simply connected source-fibers (cf. [9, 12] and the references cited there), which extends the usual equivalence between finite-dimensional Lie algebras and simply connected Lie groups. The Lie algebroids in examples (1)-(3) are all integrable: The corresponding Lie groupoids with simply connected source-fibers are the simply connected Lie group associated to the Lie algebra, the fundamental groupoid of the manifold $M$ and the monodromy groupoid associated to the foliation, respectively. However, not every Lie algebroid is integrable; for a characterization of integrability, see [2].

The algebraic analogue of a Lie algebroid is a $(k, R)$-Lie algebra, where $k$ is a field of characteristic 0 playing the role of $\mathbb{R}$ and $R$ is a unital commutative $k$ algebra playing the role of $C^{\infty}(M)$. A $(k, R)$-Lie algebra is a Lie algebra $L$ over $k$ equipped with the structure of a unital left $R$-module and with a homomorphism of Lie algebras $\rho: L \rightarrow \operatorname{Der}_{k}(R)$ into the Lie algebra of derivations on $R$, which is a map of left $R$-modules satisfying the following Leibniz rule:

$$
[X, r Y]=r[X, Y]+\rho(X)(r) Y
$$

for any $X, Y \in L$ and $r \in R$. One writes $\rho(X)(r)=X(r)$. The $(k, R)$-Lie algebras form a category

$$
(k, R) \text {-LieAlgebras }
$$

where a morphism $\phi: L \rightarrow L^{\prime}$ is a homomorphism of Lie algebras over $k$ as well as a map of $R$-modules which intertwines the representations, $\rho^{\prime} \circ \phi=\rho$. These 
$(k, R)$-Lie algebras were introduced and studied under different names by Herz [4, Palais [18] and Rinehart [20].

Example 1.2. (1) If $R=k$, then $\operatorname{Der}_{k}(R)=\{0\}$, and a $(k, k)$-Lie algebra is simply a Lie algebra over $k$.

(2) For arbitrary $R$, the Lie algebra $\operatorname{Der}_{k}(R)$ is itself a $(k, R)$-Lie algebra if one takes $\rho$ to be the identity map. More generally, if $A$ is a unital $k$-algebra, then $\operatorname{Der}_{k}(A)$ is a $(k, \mathrm{Z}(A))$-Lie algebra, where $\mathrm{Z}(A)$ is the center of $A$ and $\rho: \operatorname{Der}_{k}(A) \rightarrow$ $\operatorname{Der}_{k}(\mathrm{Z}(A))$ is given by restriction; see $[6$.

(3) In the special case $k=\mathbb{R}$ and $R=C^{\infty}(M)$ one has $\operatorname{Der}_{\mathbb{R}}\left(C^{\infty}(M)\right)=\mathfrak{X}(M)$ 23], while the Serre-Swan theorem implies that a unital left $C^{\infty}(M)$-module is isomorphic to the module of sections of a vector bundle over $M$ if and only if it is finitely generated and projective. In other words, Lie algebroids over $M$ are precisely the $\left(\mathbb{R}, C^{\infty}(M)\right)$-Lie algebras which are finitely generated and projective as $C^{\infty}(M)$-modules. Therefore, we shall identify the category (LieAlgebroids) ${ }_{M}$ with the corresponding full subcategory of the category $\left(\mathbb{R}, C^{\infty}(M)\right)$-LieAlgebras.

Let $L$ be an $(k, R)$-Lie algebra. The left $R$-module $R \oplus L$ has a natural Lie algebra structure, given by

$$
[(r, X),(s, Y)]=(X(s)-Y(r),[X, Y])
$$

for any $r, s \in R$ and $X, Y \in L$. Let $U(R \oplus L)$ be its universal enveloping algebra over $k$, obtained as the quotient of the tensor algebra of $R \oplus L$ over $k$ with respect to the usual ideal. Write $i: R \oplus L \rightarrow U(R \oplus L)$ for the canonical inclusion and $\bar{U}(R \oplus L)$ for the subalgebra of $U(R \oplus L)$ generated by $i(R \oplus L)$. The universal enveloping algebra of the $(k, R)$-Lie algebra $L$ (see [20]) is the quotient algebra

$$
\mathscr{U}(R, L)=\bar{U}(R \oplus L) / I
$$

over $k$, where $I$ is the two-sided ideal in $\bar{U}(R \oplus L)$ generated by the elements $i(s, 0) \cdot i(r, X)-i(s r, s X)$ for all $r, s \in R$ and $X \in L$. The natural map $\iota_{R}: R \rightarrow$ $\mathscr{U}(R, L), r \mapsto i(r, 0)+I$ is a homomorphism of unital $k$-algebras, while $\iota_{L}: L \rightarrow$ $\mathscr{U}(R, L), X \mapsto i(0, X)+I$ is a homomorphism of Lie algebras. Furthermore, we have $\iota_{R}(r) \iota_{L}(X)=\iota_{L}(r X)$ and $\left[\iota_{L}(X), \iota_{R}(r)\right]=\iota_{R}(X(r))$.

The universal enveloping algebra $\mathscr{U}(R, L)$ is characterized by the following universal property: if $A$ is any unital $k$-algebra, $\kappa_{R}: R \rightarrow A$ is a homomorphism of unital $k$-algebras and $\kappa_{L}: L \rightarrow A$ is a homomorphism of Lie algebras such that $\kappa_{R}(r) \kappa_{L}(X)=\kappa_{L}(r X)$ and $\left[\kappa_{L}(X), \kappa_{R}(r)\right]=\kappa_{R}(X(r))$ for any $r \in R$ and $X \in L$, then there exists a unique homomorphism of unital algebras $f: \mathscr{U}(R, L) \rightarrow A$ such that $f \circ \iota_{R}=\kappa_{R}$ and $f \circ \iota_{L}=\kappa_{L}$.

In particular, the universal property of $\mathscr{U}(R, L)$ implies that there exists a unique representation

$$
\varrho: \mathscr{U}(R, L) \rightarrow \operatorname{End}_{k}(R)
$$

such that $\varrho \circ \iota_{L}=\rho$ and $\varrho \circ \iota_{R}$ is the canonical representation given by the multiplication in $R$. Since the canonical representation of $R$ is faithful, we see that the map $\iota_{R}$ is injective. We shall therefore identify $\iota_{R}(R)$ with $R, \iota_{R}(r)=r$. We shall often denote $\iota_{L}(X)$ by $\iota(X)$ or simply by $X$. In this notation, the algebra $\mathscr{U}(R, L)$ is generated by elements $X \in L$ and $r \in R$, while $r \cdot X=r X$ and $[X, r]=X \cdot r-r \cdot X=X(r)$ in $\mathscr{U}(R, L)$. As a $k$-linear space, $\mathscr{U}(R, L)$ is generated by $R$ and the powers $\iota(L)^{n}, n=1,2, \ldots$ The algebra $\mathscr{U}(R, L)$ also has a natural 
filtration

$$
R=\mathscr{U}_{(0)}(R, L) \subset \mathscr{U}_{(1)}(R, L) \subset \mathscr{U}_{(2)}(R, L) \subset \cdots,
$$

where $\mathscr{U}_{(n)}(R, L)$ is spanned by $R$ and the powers $\iota(L)^{m}$, for $m=1,2, \ldots, n$. We define the associated graded algebra as

$$
\operatorname{gr}(\mathscr{U}(R, L))=\bigoplus_{n=0}^{\infty} \mathscr{U}_{(n)}(R, L) / \mathscr{U}_{(n-1)}(R, L),
$$

where we take $\mathscr{U}_{(-1)}(R, L)=\{0\}$. It is a commutative unital algebra over $R$.

Definition 1.3. Let $\mathfrak{g}$ be a Lie algebroid over $M$. The universal enveloping algebra $\mathscr{U}(\mathfrak{g})$ of $\mathfrak{g}$ is the universal enveloping algebra of the underlying $\left(\mathbb{R}, C^{\infty}(M)\right.$ )-Lie algebra $\Gamma \mathfrak{g}$,

$$
\mathscr{U}(\mathfrak{g})=\mathscr{U}\left(C^{\infty}(M), \Gamma \mathfrak{g}\right) .
$$

Example 1.4. (1) Let $V$ be a left $R$-module. With zero bracket and representation, $V$ is a $(k, R)$-Lie algebra. The corresponding universal enveloping algebra $\mathscr{U}(R, V)$ is in this case the symmetric algebra $S_{R}(V)$ (see the appendix below).

(2) For any Lie algebra over $k$, the universal enveloping algebra $\mathscr{U}(k, L)$ is the classical universal enveloping algebra $U(L)$ of $L$.

(3) If $G$ is a Lie groupoid over $M$ and $\mathfrak{g}$ its Lie algebroid, then the algebra of right invariant tangential differential operators on $G$ is a concrete model for the universal enveloping algebra $\mathscr{U}(\mathfrak{g})$ (see [17]).

As for the classical universal enveloping algebra of a Lie algebra, there is a Poincaré-Birkhoff-Witt theorem for the universal enveloping algebra of a $(k, R)$-Lie algebra 20]: if the $(k, R)$-Lie algebra $L$ is projective as a left $R$-module, then the natural map $\theta: S_{R}(L) \rightarrow \operatorname{gr}(\mathscr{U}(R, L))$ is an isomorphism of algebras. In particular, this implies that $\iota_{L}: L \rightarrow \mathscr{U}(R, L)$ is injective in this case.

\section{2. $R / k$-BIALGEBRAS}

The universal enveloping algebra of a Lie algebra is a Hopf algebra, as is the group ring of a discrete group. In this section we will identify the algebraic structure common to the universal enveloping algebra of a Lie algebroid and the convolution algebra of an étale groupoid as that of an $R / k$-bialgebra. The definition of this structure goes back to [15, 16, 22, 24, 25. Similar structures have occurred in the literature under various names such as quantum groupoids or Hopf algebroids (in the presence of an antipode of some kind); see e.g. 3, [5, 8, 10, 13, 14, 26, and the references cited there.

Let $R$ be a unital commutative $k$-algebra as before. All modules considered will be unital left $R$-modules, and $\otimes_{R}$ will always denote the tensor product of left $R$ modules. Suppose that $A$ is a unital $k$-algebra which extends $R$, i.e. such that $R$ is a unital subalgebra of $A$. In particular, $A$ is an $R$ - $R$-bimodule. The left $R$-module $A \otimes_{R} A$ (tensor product of $A$, viewed as a left $R$-module, with itself) is also a right $R$-module in two ways. Observe that $A \otimes_{R} A$ is not necessarily an algebra in a natural way unless $R$ lies in the centre of $A$. Following [5], we write $A \bar{\otimes}_{R} A$ for the submodule of $A \otimes_{R} A$ given by the kernel of the map $\vartheta$,

$$
0 \longrightarrow A \bar{\otimes}_{R} A \longrightarrow A \otimes_{R} A \stackrel{\vartheta}{\longrightarrow} \operatorname{Hom}_{k}\left(R, A \otimes_{R} A\right),
$$


defined by $\vartheta(a \otimes b)(r)=a r \otimes b-a \otimes b r$. (Sweedler [22] denotes this submodule by $A \times_{R} A$.) The $R$-module $A \bar{\otimes}_{R} A$ has a natural $k$-algebra structure. If $R$ is contained in the centre of $A$, then $A \bar{\otimes}_{R} A=A \otimes_{R} A$.

Definition 2.1. An $R / k$-bialgebra is a unital $k$-algebra $A$ which extends $R$, with a compatible structure of a cocommutative coalgebra in the category of left $R$ modules. If we denote the comultiplication by $\Delta: A \rightarrow A \otimes_{R} A$ and the counit by $\epsilon: A \rightarrow R$, the compatibility conditions are

(i) $\Delta(A) \subset A \bar{\otimes}_{R} A$,

(ii) $\epsilon(1)=1$,

(iii) $\Delta(1)=1 \otimes 1$,

(iv) $\epsilon(a b)=\epsilon(a \epsilon(b))$, and

(v) $\Delta(a b)=\Delta(a) \Delta(b)$

for any $a, b \in A$.

Observe that condition (v) makes sense because of (i) and the fact that $A \bar{\otimes}_{R} A$ is a $k$-algebra. Note, however, that (iv) does not express that $\epsilon$ is an algebra map. The $R / k$-bialgebras form a category

$$
R / k \text {-BiAlgebras , }
$$

in which a morphism $f: A \rightarrow B$ is a map which is at the same time a homomorphism of $k$-algebras with unit and a homomorphism of coalgebras in the category of left $R$-modules.

Example 2.2. (1) If a unital $k$-algebra $A$ extends $R$ such that $R$ lies in the centre of $A$, an $R / k$-bialgebra structure on $A$ is the same as an ordinary $R$-bialgebra structure on $A$.

(2) Let $\mathscr{U}(R, L)$ be the universal enveloping algebra of a $(k, R)$-Lie algebra $L$ (Section 11). The universal property implies that there exists a unique homomorphism of algebras

$$
\Delta: \mathscr{U}(R, L) \rightarrow \mathscr{U}(R, L) \bar{\otimes}_{R} \mathscr{U}(R, L) \subset \mathscr{U}(R, L) \otimes_{R} \mathscr{U}(R, L)
$$

such that $\Delta(r)=1 \otimes r=r \otimes 1$ and $\Delta(X)=1 \otimes X+X \otimes 1$ for any $r \in R$ and $X \in L$. With the counit $\epsilon: \mathscr{U}(R, L) \rightarrow R$ given by

$$
\epsilon(u)=\varrho(u)(1)
$$

one can check that $\mathscr{U}(R, L)$ is an $R / k$-bialgebra. Furthermore, a morphism of $(k, R)$-Lie algebras $\phi: L \rightarrow L^{\prime}$ induces, by the universal property, a morphism of $R / k$-bialgebras $\mathscr{U}(R, \phi): \mathscr{U}(R, L) \rightarrow \mathscr{U}\left(R, L^{\prime}\right)$, and this gives a functor

$$
\mathscr{U}:(k, R) \text {-LieAlgebras } \rightarrow R / k \text {-BiAlgebras . }
$$

(3) The convolution algebra of smooth functions with compact support on an étale Lie groupoid $G$ over a compact manifold $M$ is a $C^{\infty}(M) / \mathbb{R}$-bialgebra (see [13, 14]).

Let $A$ be an $R / k$-bialgebra. Then $A$ splits as an $R$-module as

$$
A=R \oplus \bar{A},
$$

where $\bar{A}=\operatorname{ker} \epsilon$. The $R$-submodule $\bar{A}$ is a subalgebra of $A$ and carries a cocommutative coassociative coproduct $\bar{\Delta}: \bar{A} \rightarrow \bar{A} \otimes_{R} \bar{A}$, defined by

$$
\bar{\Delta}(a)=\Delta(a)-a \otimes 1-1 \otimes a .
$$


The $R / k$-bialgebra $A$ can be reconstructed from $\bar{A}, \bar{\Delta}$ and the multiplication on $\bar{A}$.

The $R$-module $\bar{A}$ has a filtration

$$
\{0\}=\bar{A}_{0} \subset \bar{A}_{1} \subset \bar{A}_{2} \subset \cdots
$$

with $\bar{A}_{n}=\operatorname{ker} \bar{\Delta}^{(n)}$, where $\bar{\Delta}^{(n)}$ denotes the iterated coproduct $\bar{A} \rightarrow \bar{A} \otimes_{R} \cdots \otimes_{R} \bar{A}$ ( $n+1$ copies). We refer to this filtration as the primitive filtration of $\bar{A}$, and we also write $A_{n}=R \oplus \bar{A}_{n}(n \geq 0)$. The submodule $\bar{A}_{1}$ is called the submodule of primitive elements and is also denoted by $\mathscr{P}(A)$. We observe that $\mathscr{P}(A)$ is a $(k, R)$ Lie algebra. Its Lie bracket is given by the commutator in $A$, and its representation $\rho: \mathscr{P}(A) \rightarrow \operatorname{Der}_{k}(R)$ is given by $\rho(a)(r)=\epsilon(a r)$. Indeed, note that

$$
\begin{aligned}
a r & =(\epsilon \otimes 1)(\Delta(a r)) \\
& =(\epsilon \otimes 1)(\Delta(a) \Delta(r)) \\
& =(\epsilon \otimes 1)((a \otimes 1+1 \otimes a)(r \otimes 1)) \\
& =(\epsilon \otimes 1)(a r \otimes 1+r \otimes a) \\
& =\epsilon(a r)+r a,
\end{aligned}
$$

and from this it follows easily that $\rho$ is an $R$-linear homomorphism of Lie algebras.

We call $A$ (or $\bar{A}$ ) cocomplete if $A=\bigcup_{n=0}^{\infty} A_{n}$ (or $\bar{A}=\bigcup_{n=0}^{\infty} \bar{A}_{n}$ ). The $R / k$ bialgebra $A$ is graded projective if each of the subquotients $A_{n+1} / A_{n}=\bar{A}_{n+1} / \bar{A}_{n}$ is a projective $R$-module. We say that $A$ is of finite type if $\mathscr{P}(A)$ is finitely generated as an $R$-module. We will use these notions in several theorems stated below.

Example 2.3. It follows from the Poincaré-Birkhoff-Witt theorem that the primitive filtration of the universal enveloping algebra $\mathscr{U}(R, L)$, associated to a $(k, R)$-Lie algebra $L$, coincides with its natural filtration if $L$ is projective as a left $R$-module. Furthermore, the universal enveloping algebra $\mathscr{U}(R, L)$ is in this case cocomplete and graded projective. Finally, the universal enveloping algebra of a Lie algebroid is cocomplete, graded projective and of finite type.

Remark 2.4. Let $A$ be a cocomplete graded projective $R / k$-bialgebra. In particular, this implies that $A$ and all $A_{n}$ are projective $R$-modules. We write $\operatorname{gr}(\bar{A})=$ $\bigoplus_{n=1}^{\infty} \operatorname{gr}_{n}(\bar{A})$, where $\operatorname{gr}_{n}(\bar{A})=\bar{A}_{n} / \bar{A}_{n-1}$. There is a cocommutative coassociative comultiplication $\bar{\Delta}^{\mathrm{gr}}$ on $\operatorname{gr}(\bar{A})$, induced by $\bar{\Delta}$, such that

$$
\bar{\Delta}^{\operatorname{gr}}\left(\operatorname{gr}_{n}(\bar{A})\right) \subset \bigoplus_{p+q=n} \operatorname{gr}_{p}(\bar{A}) \otimes \operatorname{gr}_{q}(\bar{A})
$$

and $\operatorname{ker}\left(\bar{\Delta}^{\mathrm{gr}}\right)=\operatorname{gr}_{1}(\bar{A})$. Furthermore, the non-counital coalgebra $\operatorname{gr}(\bar{A})$ is cocomplete, i.e. $\bigcup_{n=1}^{\infty} \operatorname{ker}\left(\left(\bar{\Delta}^{\mathrm{gr}}\right)^{(n)}\right)=\operatorname{gr}(\bar{A})$. Note that any morphism $f: A \rightarrow B$ of cocomplete graded projective $R / k$-bialgebras induces a morphism of non-counital coalgebras $\operatorname{gr}(\bar{f}): \operatorname{gr}(\bar{A}) \rightarrow \operatorname{gr}(\bar{B})$.

\section{A Cartier-Milnor-Moore theorem}

We have already seen that the universal enveloping algebra construction defines a functor

$$
\mathscr{U}:(k, R) \text {-LieAlgebras } \rightarrow R / k \text {-BiAlgebras . }
$$

In the other direction, there is a functor

$$
\mathscr{P}: R / k \text {-BiAlgebras } \rightarrow(k, R) \text {-LieAlgebras }
$$


which assigns to an $R / k$-bialgebra $A$ its $(k, R)$-Lie algebra $\mathscr{P}(A)$ of primitive elements.

Theorem 3.1. (i) The functor $\mathscr{U}$ is left adjoint to $\mathscr{P}$.

(ii) The functors $\mathscr{U}$ and $\mathscr{P}$ restrict to an equivalence between the full subcategory of $(k, R)$-Lie algebras which are projective as left $R$-modules and that of cocomplete graded projective $R / k$-bialgebras.

(iii) In the special case $k=\mathbb{R}$ and $R=C^{\infty}(M)$, the functors $\mathscr{U}$ and $\mathscr{P}$ restrict to an equivalence between the category (LieAlgebroids) ${ }_{M}$ of Lie algebroids over $M$ and that of cocomplete graded projective $C^{\infty}(M) / \mathbb{R}$-bialgebras of finite type.

The second part of the theorem was proved by Nichols [15] in the special case where $R$ is a field extension of $k$, with a different proof. The second part of the theorem in particular implies the following property of the counit of the adjunction, which is an analogue of the Cartier-Milnor-Moore theorem for Hopf algebras:

Corollary 3.2. Let $A$ be a cocomplete graded projective $R / k$-bialgebra. Then there is a canonical isomorphism of $R / k$-bialgebras $A \cong \mathscr{U}(R, \mathscr{P}(A))$.

Remark 3.3. In particular, if $A$ is a cocomplete graded projective $C^{\infty}(M) / \mathbb{R}$ bialgebra of finite type, then, up to an isomorphism, it is the universal enveloping algebra of a unique Lie algebroid over $M$.

Proof of Theorem 3.1. For a $(k, R)$-Lie algebra $L$, the canonical map $L \rightarrow \mathscr{U}(R, L)$ clearly lands in the submodule of primitive elements, and this defines the unit of the adjunction,

$$
\alpha_{L}: L \rightarrow \mathscr{P}(\mathscr{U}(R, L))
$$

For an $R / k$-bialgebra $A$, the inclusion $\mathscr{P}(A) \rightarrow A$ induces, by the universal property of the universal enveloping algebra, a canonical algebra map

$$
\beta_{A}: \mathscr{U}(R, \mathscr{P}(A)) \rightarrow A,
$$

which in fact is clearly a map of $R / k$-bialgebras. This defines the counit of the adjunction.

The first part of the theorem states that these two maps satisfy the triangular identities

and

$$
\mathscr{P}\left(\beta_{A}\right) \circ \alpha_{\mathscr{P}(A)}=\operatorname{id}_{\mathscr{P}(A)}
$$

$$
\beta_{\mathscr{U}(R, L)} \circ \mathscr{U}\left(R, \alpha_{L}\right)=\operatorname{id}_{\mathscr{U}(R, L)} .
$$

These both hold by the (uniqueness part of the) universal property of the universal enveloping algebra.

If $L$ is projective as a left $R$-module, then the Poincaré-Birkhoff-Witt theorem implies that $\mathscr{U}(R, L)$ is graded projective and cocomplete (because the natural and primitive filtrations coincide) and that $\alpha_{L}$ is an isomorphism. For a graded projective cocomplete $R / k$-bialgebra $A$, the $R$-module $\mathscr{P}(A)$ is obviously projective, and it remains to show that in this case the map $\beta=\beta_{A}$ is an isomorphism.

It suffices to prove that the map of reduced non-counital coalgebras

$$
\bar{\beta}: \overline{\mathscr{U}}(R, \mathscr{P}(A)) \rightarrow \bar{A}
$$

is an isomorphism. For this, in turn, it is enough to show that the induced map of non-counital coalgebras

$$
\operatorname{gr}(\bar{\beta}): \operatorname{gr}(\overline{\mathscr{U}}(R, \mathscr{P}(A))) \rightarrow \operatorname{gr}(\bar{A})
$$


is an isomorphism. The projection $\operatorname{gr}(\bar{A}) \rightarrow \operatorname{gr}_{1}(\bar{A})=\mathscr{P}(A)$ induces a map of noncounital coalgebras $\gamma: \operatorname{gr}(\bar{A}) \rightarrow \bar{S}_{R}(\mathscr{P}(A))$ by the universal property of $\bar{S}_{R}(\mathscr{P}(A))$ (Proposition A.2). Now consider the diagram

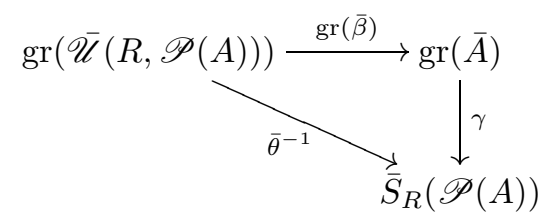

where $\bar{\theta}^{-1}$ is the inverse of the Poincaré-Birkhoff-Witt isomorphism $\theta$ (Section 1) restricted to $\bar{S}_{R}(\mathscr{P}(A))$. All maps in the diagram are maps of non-counital coalgebras. Thus, to see that the diagram commutes, it suffices (again by the universal property of $\bar{S}_{R}(\mathscr{P}(A))$ stated in Proposition A.2) that

$$
\operatorname{pr}_{1} \circ \gamma \circ \operatorname{gr}(\bar{\beta})=\operatorname{pr}_{1} \circ \bar{\theta}^{-1}
$$

for the projection $\operatorname{pr}_{1}: \bar{S}_{R}(\mathscr{P}(A)) \rightarrow \mathscr{P}(A)$, which is clear from the explicit definitions. To finish the proof, recall from Remark 2.4 that $\operatorname{gr}(\bar{A})$ is cocomplete and $\operatorname{ker}\left(\bar{\Delta}^{\mathrm{gr}}\right)=\mathscr{P}(A)$, so that by Lemma A.1 the map $\gamma$ is injective, while by the commutativity of the diagram it is also surjective. Thus $\gamma$ is an isomorphism, and hence so is $\operatorname{gr}(\bar{\beta})$.

\section{Appendix A. Cocommutative non-Counital COALGebras}

The main goal of this appendix is to prove some elementary properties of cocommutative non-counital coalgebras over a ring, in particular the universal property of the symmetric coalgebra. Although these results are presumably well-known, they are usually stated in the literature in the context of coalgebras over a field (see e.g. [1, 11, 19, 21]).

As before, $k$ denotes a field of characteristic 0 and $R$ a unital commutative $k$-algebra. A cocommutative non-counital coalgebra over $R$ is an $R$-module $C$, together with a cocommutative coassociative comultiplication $\delta: C \rightarrow C \otimes_{R} C$. (Note that we do not assume that $C$ has a counit.) With the obvious maps, these cocommutative non-counital coalgebras over $R$ form a category. For example, for an $R / k$-bialgebra $A$, the pair $(\bar{A}, \bar{\Delta})$ is an object of this category.

As already discussed in this special case, any cocommutative non-counital coalgebra $(C, \delta)$ carries a primitive filtration

$$
\{0\}=C_{0} \subset C_{1} \subset C_{2} \subset \cdots,
$$

where $C_{n}$ is the kernel of the iterated coproduct $\delta^{(n)}: C \rightarrow C \otimes_{R} \cdots \otimes_{R} C(n+1$ copies). We say that $C$ is cocomplete if $C=\bigcup_{n=0}^{\infty} C_{n}$. (In the literature, one also uses connected or conilpotent.) If $C$ is cocomplete and $C_{n}$ are all flat $R$-modules, then $C$ is a flat $R$-module as well. Notice that in this case $\delta: C \rightarrow C \otimes_{R} C$ maps each $C_{n+1}$ into $C_{n} \otimes_{R} C_{n}$.

Lemma A.1. Let $f: C \rightarrow D$ be a morphism of cocomplete cocommutative noncounital coalgebras over $R$, and assume that all the submodules $C_{n}, D_{n}$ are flat. If $f_{1}=\left.f\right|_{C_{1}}: C_{1} \rightarrow D_{1}$ is injective, then so is $f: C \rightarrow D$. 
Proof. We prove by induction that $f_{n}=\left.f\right|_{C_{n}}: C_{n} \rightarrow D_{n}$ is injective. Assuming this has been proved for $f_{n}$, injectivity of $f_{n+1}$ follows from the diagram

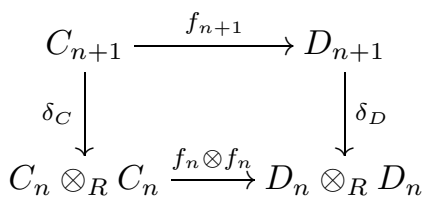

since the map at the bottom is injective by the flatness assumption. Indeed, we have $\operatorname{ker}\left(f_{n+1}\right) \subset \operatorname{ker}\left(\delta_{C}\right)=C_{1}$ and $C_{1} \cap \operatorname{ker}(f)=\{0\}$ by assumption.

Let $V$ be an $R$-module. We denote by $S_{R}(V)$ the symmetric algebra on $V$. It is a graded algebra, where $S_{R}^{n}(V)$ is the space $\left(V \otimes_{R} \cdots \otimes_{R} V\right)_{\Sigma_{n}}$ of coinvariants of the $n$-fold tensor product. The algebra $S_{R}(V)$ is the free commutative unital $R$-algebra on $V$. Therefore, the map $\Delta: V \rightarrow S_{R}(V) \otimes_{R} S_{R}(V)$, given by $\Delta(v)=1 \otimes v+v \otimes 1$, extends uniquely to a unital algebra homomorphism $\Delta: S_{R}(V) \rightarrow S_{R}(V) \otimes_{R} S_{R}(V)$, giving $S_{R}(V)$ the structure of a commutative and cocommutative bialgebra over $R$. (In fact, this is the $R / k$-bialgebra $\mathscr{U}(R, L)$, where $L$ is $V$ viewed as a $(k, R)$-Lie algebra with zero bracket and representation.)

The bialgebra $S_{R}(V)$ also has a universal property as a coalgebra, most easily stated in terms of the $R$-module $\bar{S}_{R}(V)=\bigoplus_{n=1}^{\infty} S_{R}^{n}(V)$, which is a cocomplete cocommutative non-counital $R$-algebra with coproduct $\bar{\Delta}(c)=\Delta(c)-1 \otimes c-c \otimes 1$.

Proposition A.2. Let $(C, \delta)$ be a cocomplete cocommutative non-counital coalgebra over $R$ and $V$ be an $R$-module. Then any morphism of $R$-modules $C \rightarrow V$ is the first component of a unique homomorphism $C \rightarrow \bar{S}_{R}(V)=\bigoplus_{n=1}^{\infty} S_{R}^{n}(V)$ of non-counital coalgebras over $R$.

Proof. Let $g_{1}: C \rightarrow V=S_{R}^{1}(V)$ be an $R$-linear map. We will define a map $g_{n}: C \rightarrow S_{R}^{n}(V)$, for each $n \geq 2$, in such a way that these together form a coalgebra map $g=\left(g_{n}\right): C \rightarrow \bar{S}_{R}(V)=\bigoplus_{n=1}^{\infty} S_{R}^{n}(V)$. Note that for $g$ to be a coalgebra map, there can be at most one such $g_{n}$, since it will have to make the diagram

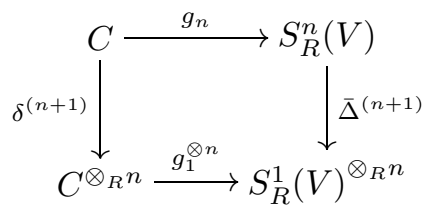

commute. In fact, we can use this diagram to define $g_{n}$, because $C$ is cocommutative and $\bar{\Delta}^{(n+1)}$ gives an isomorphism between $S_{R}^{n}(V)$ and the subspace of $\Sigma_{n}$-invariants of $S_{R}^{1}(V)^{\otimes_{R} n}$. (Here we used the assumption that the characteristic of $k$ is 0 .)

The cocompleteness of $C$ implies that $g$ is well defined. To see that $g$ is indeed a map of coalgebras, we show by induction on $n$ that the diagram

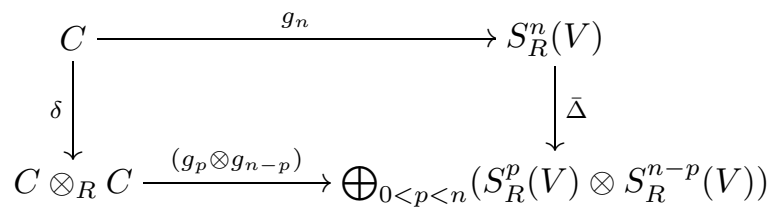

commutes. For $n=1$ there is nothing to prove, and for $n=2$ this holds by definition of $g_{2}$. Take $n>2$, and suppose that the commutativity of (1) has been 
proved for all $m<n$. Now consider the diagram

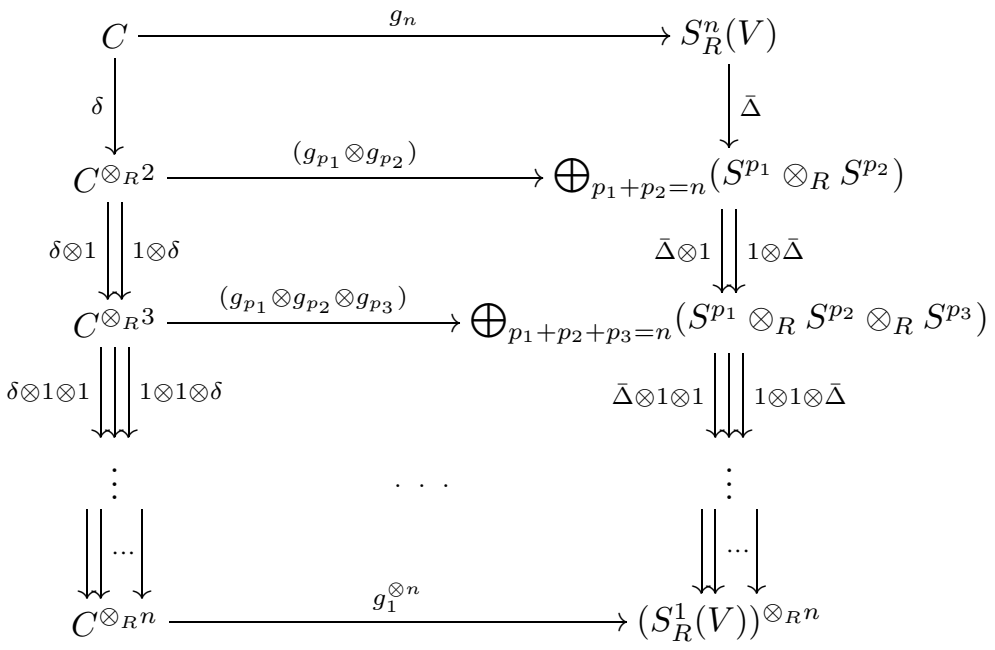

where all $p_{i} \geq 1$ and $S^{p_{i}}=S_{R}^{p_{i}}(V)$. Our aim is to prove that the top square in (2) commutes. By the induction hypothesis, the two parallel squares at the second level commute, as do the parallel squares at each lower level. Furthermore, the outer square commutes by the definition of $g_{n}$, as the vertical compositions $C \rightarrow C^{\otimes_{R} n}$ all agree and define $\delta^{(n+1)}$, and similarly for all the vertical compositions $S_{R}^{n}(V) \rightarrow\left(S_{R}^{1}(V)\right)^{\otimes_{R} n}$. Thus, to show that the top square commutes, it suffices to prove that the joint kernel of all possible vertical compositions

$$
\bigoplus_{p_{1}+p_{2}=n}\left(S^{p_{1}} \otimes_{R} S^{p_{2}}\right) \rightarrow\left(S_{R}^{1}(V)\right)^{\otimes_{R} n}
$$

is zero, which can easily be checked (note that $\bar{\Delta}: S_{R}^{n}(V) \rightarrow \bigoplus_{p_{1}+p_{2}=n}\left(S^{p_{1}} \otimes_{R} S^{p_{2}}\right)$ is a split injective map of left $R$-modules if $n \geq 2)$.

\section{REFERENCES}

[1] P. Cartier, A primer of Hopf algebras. Frontiers in number theory, physics, and geometry. II. 537-615, Springer, Berlin, 2007. MR2290769 (2008b:16059)

[2] M. Crainic, R. L. Fernandes, Integrability of Lie brackets. Ann. of Math. 157 (2003) 575-620. MR:1973056 (2004h:58027)

[3] R. L. Grossman, R. G. Larson, Differential algebra structures on families of trees. Adv. in Appl. Math. 35 (2005) 97-119. MR2141507 (2006a:16051)

[4] J.-C. Herz, Pseudo-algèbres de Lie. I, II. C. R. Acad. Sci. Paris 236 (1953) 1935-1937, 22892291.

[5] M. Kapranov, Free Lie algebroids and the space of paths. Selecta Math. 13 (2007) 277-319. MR2361096 (2009h:53108)

[6] V. K. Kharchenko, Automorphisms and derivations of associative rings. Translated from the Russian by L. Yuzina. Mathematics and its Applications (Soviet Series), 69, Kluwer Academic Publishers Group, Dordrecht, 1991. MR 1174740 (93i:16048)

[7] J.-L. Loday, Generalized bialgebras and triples of operads. Astérisque 320 (2008). MR 2504663

[8] J.-H. Lu, Hopf algebroids and quantum groupoids. International J. Math. 7 (1996) 47-70. MR1369905 (97a:16073)

[9] K. Mackenzie. General theory of Lie groupoids and Lie algebroids. London Mathematical Society Lecture Note Series, 213, Cambridge University Press, Cambridge, 2005. MR 2157566 (2006k:58035) 
[10] G. Maltsiniotis, Groupoïdes quantiques de base non commutative. Comm. Algebra 28 (2000) 3441-3501. MR1765327 (2001f:20143)

[11] J. W. Milnor, J. C. Moore, On the structure of Hopf algebras. Ann. of Math. 81 (1965) 211-264. MR0174052(30:4259)

[12] I. Moerdijk and J. Mrčun, Introduction to foliations and Lie groupoids. Cambridge Studies in Advanced Mathematics, 91, Cambridge University Press, Cambridge, 2003. MR2012261 (2005c:58039)

[13] J. Mrčun, The Hopf algebroids of functions on étale groupoids and their principal Morita equivalence. J. Pure Appl. Algebra 160 (2001) 249-262. MR1836002 (2002h:16061)

[14] J. Mrčun, On duality between étale groupoids and Hopf algebroids. J. Pure Appl. Algebra 210 (2007) 267-282. MR2311185 (2009b:16091)

[15] W. D. Nichols, The Kostant structure theorems for $K / k$-Hopf algebras. J. Algebra 97 (1985) 313-328. MR812990 (87d:16009)

[16] W. Nichols, B. Weisfeiler, Differential formal groups of J. F. Ritt. Amer. J. Math. 104 (1982) 943-1003. MR675306 (84j:14045)

[17] V. Nistor, A. Weinstein, P. Xu, Pseudodifferential operators on differential groupoids. Pacific J. Math. 189 (1999) 117-152. MR1687747(2000c:58036)

[18] R. S. Palais, The cohomology of Lie rings. Proc. Sympos. Pure Math., Vol. III, 130-137, American Mathematical Society, Providence, RI, 1961. MR.0125867 (23:A3164)

[19] D. Quillen, Rational homotopy theory. Ann. of Math. (2) 90 (1969) 205-295. MR0258031 (41:2678)

[20] G. S. Rinehart, Differential forms on general commutative algebras. Trans. Amer. Math. Soc. 108 (1963) 195-222. MR0154906 (27:4850)

[21] M. E. Sweedler, Hopf algebras. Mathematics Lecture Note Series, W. A. Benjamin, Inc., New York, 1969. MR0252485 (40:5705)

[22] M. E. Sweedler, Groups of simple algebras. Inst. Hautes Études Sci. Publ. Math. 44 (1974) 79-189. MR0364332(51:587)

[23] F. Takens, Derivations of vector fields. Compositio Math. 26 (1973), 151-158. MR0315723 $(47: 4272)$

[24] M. Takeuchi, Groups of algebras over $A \otimes \bar{A}$. J. Math. Soc. Japan 29 (1977) 459-492. MR0506407 (58:22151)

[25] D. Winter, The structure of fields. Graduate Texts in Mathematics, 16, Springer-Verlag, New York-Heidelberg, 1974. MR0389873 (52:10703)

[26] P. Xu, Quantum groupoids and deformation quantization. C. R. Acad. Sci. Paris Sér. I Math. 326 (1998) 289-294. MR.1648433(99h:58073)

Mathematical Institute, Utrecht University, P.O. Box 80.010, 3508 TA Utrecht, The Netherlands

E-mail address: I.Moerdijk@uu.nl

Department of Mathematics, University of Ljubljana, Jadranska 19, 1000 Ljubljana, SLOVEnia

E-mail address: janez.mrcun@fmf.uni-lj.si 